\title{
Clinical Decision Support for Diabetes in Scotland: evaluation of user acceptance, clinical processes and outcomes
}

\section{NHS \\ SCOTLAND}

NT Conway*, SG Cunningham, A Emslie-Smith, A Wales, DJ Wake

Introduction

Clinician Decision Support Systems (CDSS) are associated with improved adherence to clinical guidelines in the care of those with diabetes. CDSS was implemented within the Scottish national electronic health record, SCl-Diabetes and has been live to users within NHS Tayside and Lothian since Dec'13, serving a combined diabetes population of 30,000 (see fig. 1). This study aims to describe users' attitudes and reactions to the system and to quantify impact on clinical processes and outcomes.

\section{Method}

Health care professional (HCP) opinion was sought via focus groups and questionnaires. SCI-Diabetes data were extracted for two time periods: Dec'13-Feb'14 (Ninewells hospital, Dundee) and Aug'14-Nov'14 (St John's hospital, Livingston).

SCl-Diabetes usage was quantified using HCP interaction ("mouse-clicks") and time spent within the patient record. HCP behaviour was compared between instances where CDSS messages were displayed, with instances where not (corrected for user-role, patient age, diabetes type/duration, co-morbidity and deprivation).

Case-control comparison was made to assess clinical processes and outcomes. Cases were patients whose HCP received a CDSS message during the intervention period. Controls were matched for age; sex; diabetes type and duration; BMI and clinic attendance in areas outwith the pilot. Clinical process measures were taken within 30 days of the clinic consultation and included screening for hypercholesterolaemia, kidney, foot and thyroid disease. Additional process measures were the proportion of patients newly prescribed oral hypoglycaemic agents and angiotensin converting enzyme inhibitors (ACEI) or angiotensin receptor blockers (ARB) in the month following clinic. Clinical outcomes included HbA1c; cholesterol; blood pressure, and urinary albumin/creatinine (UACR) at 1 year. Comparison was made using multivariable regression.

\section{Results}

Pre and post-intervention HCP questionnaire response rate was 57/105 (54\%) and 39/105 (37\%). Three focus groups were held ( $n=8-9 /$ group). The majority of respondents/participants had a positive or neutral response to the system. Early-adopters reported usage within clinical workflow (see box 1). CDSS messages were displayed on opening 6,665/17,280 (39\%) records. For nurses, presence of CDSS message was associated with increased SCIDiabetes usage, compared with instances where no message was displaved (median "clicks" 19(IOrange:8-37) vs. $16(7-32)$, adj.p $=0.014)$. For doctors, CDSS messages were associated with reduced time within the patient record (median duration 33 (IQrange:5-86) vs. 38(12-97) secs, adj. $p=0.032$ ).

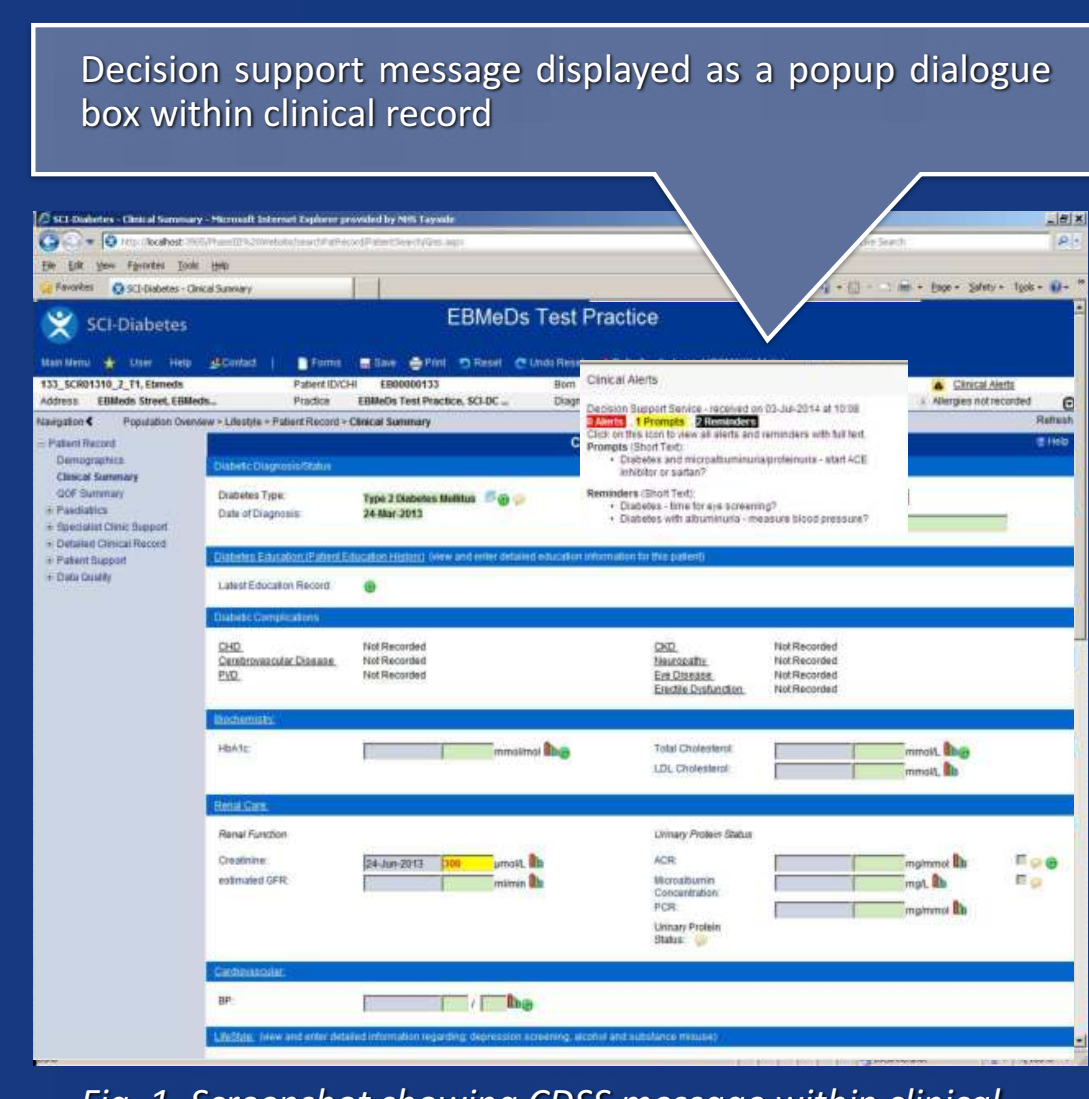

Fig. 1. Screenshot showing CDSS message within clinical record. Users navigate to message contents for further

"one of the things that I find more useful is the things that 'm not very good at . [finding] so, I'll see someone's blood pressure, and I'll know it's high, but if the computer s prompting me that they've also got microalbuminuria then it, kind of, saves me looking it up... and if it's prompting me that they've got a high cholesterol and they're not on a statin, it's, again, it's something that's just an extra prompt for things that I kind of forget." Consultant diabetelogist

Box 1. Focus group transcript

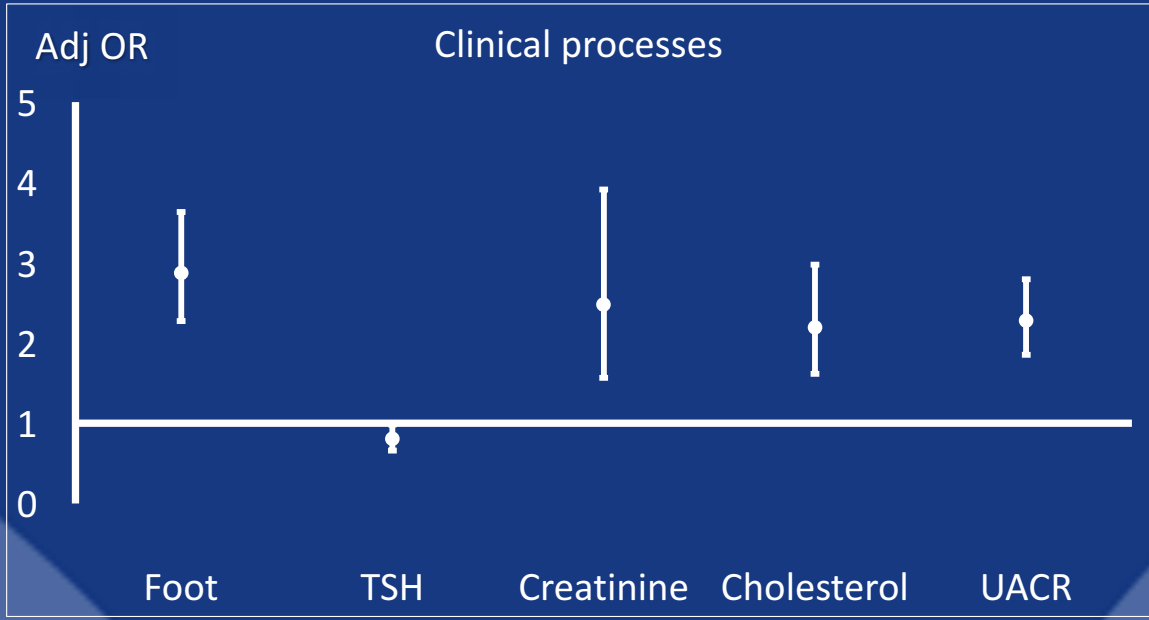

Fig. 2. Adjusted odds ratios for each of the primary outcomes Odds represent the probability of a case receiving screening for the complications of diabetes following a clinic appointment, compared with controls. Adjusted for user-role, age, diabetes type and duration, gender, socioeconomic status and comorbidity.
1,883 cases attended clinic, matched to 3,557 controls. Probability of receiving screening more than doubled for hypercholesterolaemia (adjOR 2.4, (95\%Cl: 1.6-3.0)); creatinine (2.5(1.6-3.9)); UACR (2.3(1.9$2.8)$ ); and foot screening $(2.9(2.3-3.6))$ - all $p<0.001$. Screening for hypothyroidism decreased slightly $(0.8(0.7-1.0), p=0.035)$ (see fig. 2).

Prior to the intervention, 995/1883 (52.8\%) cases and 1880/3557 (52.8\%) controls were prescribed an oral hypoglycaemic agent. Of those that were not previously on an oral hypoglycaemic agent, 15/888 (1.5\%) cases and 40/1677 (2.4\%) controls were commenced on one in the 30 days following their clinic appointment, with no significant difference noted after correcting for age, BMI, diabetes type and duration. Prior to the intervention, 1160/1,883 (61.6\%) cases and 2196/3557 (61.7\%) controls were prescribed an ACEl or ARB. Of those not previously on an ACEl or ARB, 11/723 (1.5\%) cases and 32/1361 (2.4\%) controls were commenced on one in the 30 days following their clinic appointment. There were no significant difference noted between groups after correcting for age; BMl; pre-intervention microalbuminuria or hypertension.

Of all patients whose HCP received a CDSS prompt during the intervention period (i.e. not solely within the clinic environment, $n=5,692$ ) there were small improvements in mean HbA1c compared to controls $(\mathrm{n}=10,667$ ) (baseline mean HbA1c $71.4 \mathrm{mmol} / \mathrm{mol}$ vs. 70.6 , falling by -2.3 vs. $-1.1, p=0.003$ ). Mean UACR increased in both groups but moreso in controls (baseline $8.7 \mathrm{mg} / \mathrm{mmol} \mathrm{vs}$. 9.3, increasing by $+1.6 \mathrm{vs} .+4.4$ $p=0.01$ ). Both serum cholesterol and blood pressure fell in both groups with no significant differences noted (with the exception of systolic BP - controls experienced significantly greater decrease (baseline mean SBP $137 \mathrm{mmHg}$ versus 138 , falling by -1.3 vs. $-3.3, p<0.001)$ ).

\section{Discussion/conclusion}

The CDSS was associated with improved efficiencies in working practices (dependent on role) and large improvements in guideline adherence. If replicated nationally, thousands more individuals would receive appropriate screening tests. These evidence-based, early interventions can significantly impact on costly and devastating complications such as foot ulcers, amputations, cardiovascular disease, renal failure and death. The potential benefits of this project extend beyond the Scottish diabetes population, as NHS Scotland considers how best to realise the full potential of CDSS.

*Contact: n.z.conway@dundee.ac.uk 